\title{
A Segmentation based Automated System for Brain Tumor Detection
}

\author{
Md. Sujan \\ B.Sc. Student, Dept. of CSE \\ Sylhet Engineering College \\ Sylhet, Bangladesh \\ Syed Abdullah Noman \\ B. Sc. Student, Dept. of CSE \\ Sylhet Engineering College \\ Sylhet, Bangladesh
}

\author{
Nashid Alam \\ Assistant Professor, Dept. of CSE \\ Sylhet Agricultural University \\ Sylhet, Bangladesh \\ M. Jahirul Islam, PhD, PEng \\ Professor, Dept. of CSE \\ Shahjalal University of \\ Science and Technology \\ Sylhet, Bangladesh
}

\begin{abstract}
The functionality of brain can be disrupted by brain tumor, which is an abnormal growth of tissue in brain or central spine. Due to undefined size, shape and location, detection of brain tumor from MRI (Magnetic Resonance Imaging) is a challenging and difficult task. Previous tumor segmentation methods were generally based on intensity enhancement techniques on T1-weighted image, which was appeared with gadolinium contrast agent on strictly uniform intensity patterns. This paper presents a new method based on Thresholding along with morphological image analysis techniques to detect brain tumor from MRI image. The image was first converted to grayscale and then noises were removed by applying different filtering techniques. The grayscale image was then converted to binary image adding 0.3 with the Otsu's threshold value to perfectly segment the tumor region. Afterwards, morphological operations were performed to detect the tumor that contains the brightest part of the MRI. The method suggested for detection was tested over 72 FLAIR images of 72 patients taken from BRATS Brain Tumor database, out of which the proposed algorithm was able to detect tumor from 61 images successfully. Experimental result showed an accuracy rate of $84.72 \%$ in detecting 61 patients Brain Tumor which is very much promising compares to other existing method.
\end{abstract}

\section{General Terms}

Image Segmentation, Medical Image Analysis, Digital Image Processing.

\section{Keywords}

Brain tumor, MRI, FLAIR, BRATS, Segmentation, Image morphology.

\section{INTRODUCTION}

Abnormal growth of cells inside the brain is referred as brain tumor. The normal activity of brain is obstructed by it which is why early detection of tumor is crucial. Segmentation of brain tumor is challenging as it has no predefined location, shape and size. It is also difficult, as more than one region of tumor can be found in a single MRI slice. MRI image has 4 general types of imaging modalities: T1 (T1 weighted image), T2 (T2 weighted image), T1C (T1 weighted MRI after administration of contrast media) and FLAIR (Fluid Attenuated Inversion-Recovery image). Signal enhancements of tumors were shown mostly in T1C images after administration of contrast agent [1]. Using FLAIR image allows us better detection rate of brain tumor as bright signals of the Cerebrospinal fluid (CSF) are suppressed [1]. Tumor appears to be in the brightest region in grayscale image. MRI image is better than CT and ultrasound image, though noise appears in it [2]. To accurately detect tumor, some preprocessing steps are required. Various methods have been proposed to detect Brain Tumor. A method to detect macroscopic tumor is developed by Sachin[3] using Segmentation based on the symmetric character of MRI. Pratik et al [4] used connected component analysis to detect brain tumor. Histogram and thresholding based method was proposed by Kowari and $\operatorname{Yadav}(2012)[5]$ which requires manual image cropping so that the exact result of the tumor region is not obtained. K-means Clustering technique combining with Perona-Malik Anisotropic Diffusion model was used by Ahmed and Mohamad [6] for the detection of brain tumor. Nagalkar and Asole(2012)[7] proposed brain tumor detection using soft computing method based on parameter (pixel value in grayscale image) extraction of tumor region which can cause false detection in seeing scan. Vasuda and Satheesh(2010) [8], proposed a technique to detect tumors from MR image using fuzzy C-means clustering technique but huge computational time is the major drawback of this algorithm. Classifiers are also known as supervised methods since they require training data that are manually segmented and then used as references for automatic segmentation of new data. Moreover using of the same training data for classifying a large number of images, may lead to biased result. Prastawa et al.(2004)[9] carried out outlier detection followed by geometric and spatial constraints. However, use of template limits the efficiency of the results to the accuracy of the template/atlas and so makes it non-real time in nature. Also, use of T2 channel for separation of tumor and edema is restricted to specific cases having distinct non-overlapping tumor-edema intensities. One-Class support vector machine (SVM) technique was used by Zhou et al.(2005)[10] for tumor image segmentation. Symmetry integration in several steps associated with segmentation, clustering and classification based technique was used by Sun et al.(2009) [11].However, uses of small and unstructured dataset restricts the generality and clinical applicability. Swe and Khaing [12], proposed a method using Watershed Segmentation, to calculate tumor affected area. ,in their approach, the location of the tumor was identical but there were no information about the number of image tested and accuracy rate. In this paper, a color based segmentation technique is developed using $\mathrm{k}$-means clustering approach for the detection of brain tumor. The image is first 
converted to $\mathrm{L}^{*} \mathrm{a} * \mathrm{~b}$ color space and then are classified to $\mathrm{a} * \mathrm{~b} *$ space using k-means clustering approach. The clusters are then converted to binary image and then the blob containing the tumor was found using the morphological analysis of tumor region. Experimental results showed an accuracy of $52.78 \%$ in detecting tumor region. Due to the low accuracy rate of this approach, we developed a new method based on thresholding and morphological processing. The image are converted to grayscale using MATLAB function im2gray() and after removing noise by median filtering, binarization is carried out using OTSU's[16] thresholding and morphological erosion, dilation, hole filling and biggest blob opening were applied for better segmentation and detection of tumor blob accurately where experimental results showed higher accuracy than previous obtaining $84.72 \%$.Both methods are developed using FLAIR modalities of MRI as bright signal of CSF( Cerebrospinal fluid )is suppressed in it ,from BRATS brain tumor database and detected tumor blob automatically.

\section{METHOD AND MATERIALS}

The research was implemented using MATLAB 2014, and BRATS brain tumor database from Midas Digital Archive [13], where four types of image are available for each patient: T1, T2, FLAIR and T1C. The image consists of multi modal MRI scans of different patients both in low-grade and highgrade active tumor and edema. All images are skull stripped and interpolated to $1 \mathrm{~mm}$ isotropic resolution. Patients with high and low grade gliomas have file name "BRATS HG" and "BRATS_LG" respectively [14].All images are stored in signed 16-bit integers. The database also contains simulated images having file name as "SimBRATS" which is stored as unsigned 16 bit integers. A total number of 72 FLAIR sliced MRI image of different patients' was used in our study. The said database contains 3D image in .mha format with a dimension of $160 * 216 * 176$. The .mha file was converted to .png format by using Look3D [15], where the 3-D image can be navigated in three different orthogonal views and the intensity value of each voxel locations were founded by moving the mouse pointer over any of these three views. Conversion of $2 \mathrm{D}$ image from $3 \mathrm{D}$ was done by removing the slider and point navigator of "Z" orthogonal views from Look3D viewer. The converted 2D image was saved in .png format with a dimension of $252 * 252$ and bit depth of 32 . In Fig. 2(a) shows 3D view of the image BRATS_HG001_FLAIR and Fig. 2(b) shows 2D image after converting it.

\subsection{Color Based Segmentation with K- means Clustering (CBS)}

Segmentation of tumor region was carried out using Lab Color space defined by the CIE Lab.The input images were converted to $\mathrm{L}^{*} \mathrm{a} \mathrm{b}^{*}$ color space based on one channel for Luminance (lightness) (L) and two color channels ( $\mathrm{a}$ and $\mathrm{b}$ ) using MATLAB function "makecform ('srgb2lab')" and "applycform". The color transformation structures were created by makecform ('srgb2lab') specified by 'srgb2lab'which converts the srgb image to $\mathrm{L}^{*} \mathrm{a} * \mathrm{~b} *$ color space applycform () function was used to pass the color transformation structure as an argument for performing the transformation. Conversion of $\mathrm{L}^{*} \mathrm{a} * \mathrm{~b}$ color space were shown in Fig. 4(b). The lab color space images were then classified into ' $a * b$ ' Space Using K-Means Clustering where axis a extends from green $(-a)$ to red $(+a)$ channel and the axis $b$ from blue (-b) to yellow $(+b)$ channel. Fig. 4(c) shows the classified $a^{*} b^{*}$ space image For avoiding local minima, the clustering were repeated for 3 times. We used $\mathrm{k}=3$ in our method which separates the labeled image into 3 clusters showing the tumor region in one .Optimal selection of number of cluster were shown in table 1 when $\mathrm{k}=3$. The image was then converted into binary using MATLAB function "im2bw". Morphological erosion with structuring element [1, 1] was then applied for accurate segmentation of tumor and then holes were filled showing in Fig. 4(f) and 4(g) respectively. Erosion with other structuring element rather than this can cause false segmentation as extra region appears with tumor in this method The blob was separated after opening the biggest blob by removing pixels smaller than 250 from the hole filled image which provides the exact segmentation of tumor region. Tumor in blob is detected by invoking MATLAB function: regionProps (binaryImage,'BoundingBox') to find object properties in the binary image. Fig. 4(h) shows biggest blob opening. A rectangle was drawn using the position from BoundingBox which was shown in Fig. 4(i). when the images consisting similarity of regions intensity lab color space performs better than other existing method and especially efficient when images have non uniform illumination such as shade but if the intensity of the color lies close to white or black then performs little for segmentation. Procedural diagram of CBS were shown in Fig. 1 and Fig. 3 shows the Procedural output of Color based segmentation using k-means clustering on BRATS_HG001_FLAIR image.

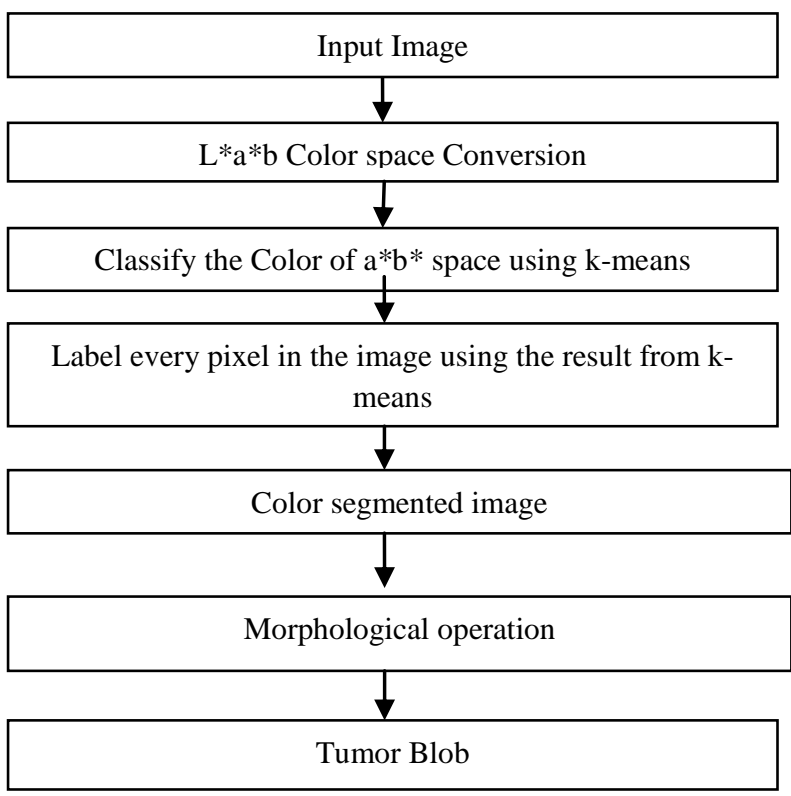

Fig 1: Diagram of Color based image segmentation using K-means clustering (CBS) 

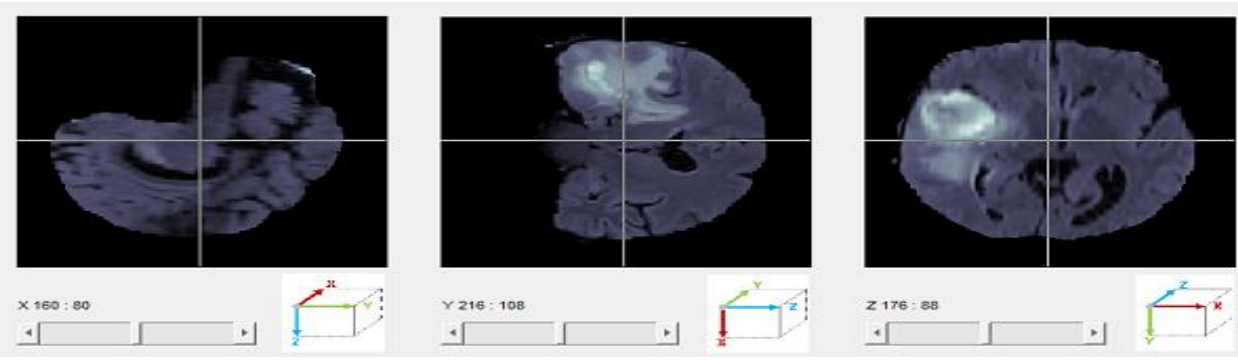

(a)3D view opened by Look3 $\mathrm{D}$ viewer

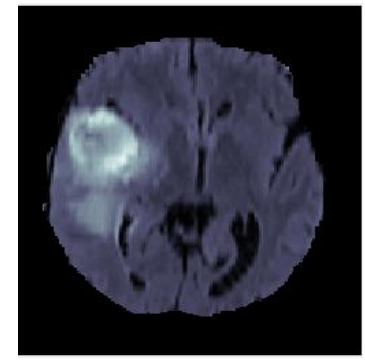

(b)Converted 2D view

Fig 2: 3D (.mha) image to 2D (.png) image conversion using Look3D

Detection of tumor was done $52.78 \%$ accurately applying this method which detects 38 patients tumor from 72 patients MRI image. Finally a new method Thresholding and Morphological Processing (TMP) were developed for brain tumor detection as the accuracy rate was very poor for Color based Segmentation using k-means clustering (CBS) method.

Algorithm-01: Color based image segmentation using kmeans clustering (CBS)

Input: BRATS_HG_0001_FLAIR MRI

Begin:

\section{Load the input MRI}

2. Convert the input image into $\mathrm{L}^{*} \mathrm{a} * \mathrm{~b} *$ color space

3. Classify the Colors in $\mathrm{a}^{*} \mathrm{~b} *$ Space Using K-Means

Clustering

4. Label Every Pixel in the Image Using the Results from K-means

5. Convert the output into binary image.

6 . Erode the binary image with Structuring element $[1 ; 1]$

7. After erosion fill the holes.

8. Open the biggest region of hole filled image

9. Detect Tumor with blob after finding 4 points of rectangle

End;

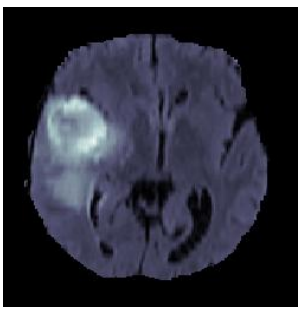

a)Input image

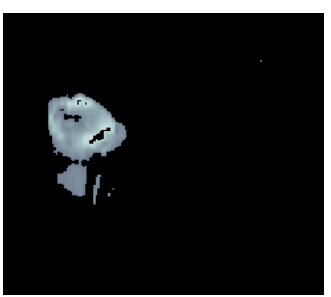

d)Tumor in cluster

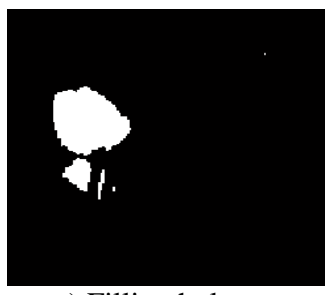

g) Filling holes

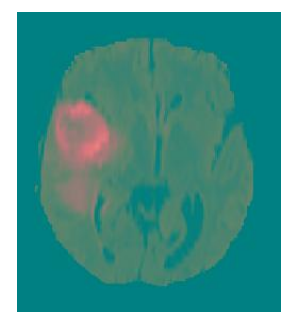

b) $\mathrm{L}^{*} \mathrm{a} * \mathrm{~b}$ color space

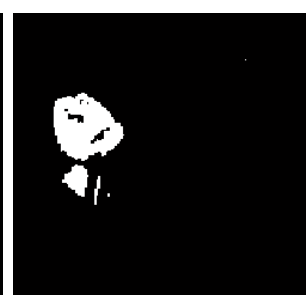

e)Binary image

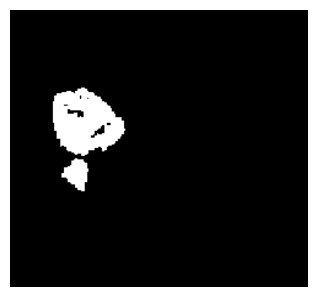

h) Biggest blob opening

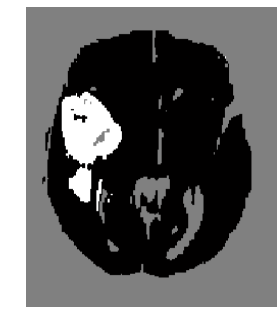

c)a* $b^{*}$ space after $\mathrm{k}$ means clustering

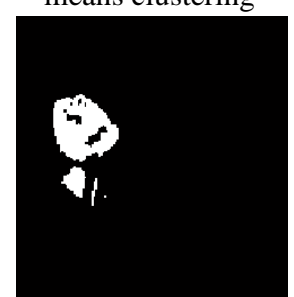

f)Morphological eroding

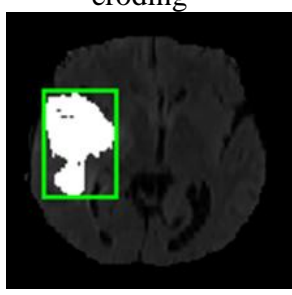

i) Tumor blob
Fig 3: Procedurals output of Color based segmentation usingKmeans clustering on BRATS_HG0001_FLAIR image

\subsection{Thresholding and Morphological Processing (TMP)}

In this method, the input image was converted to grayscale and noise was removed by performing median filtering operation. Then the image is enhanced by adding value 25 with entire image pixels that makes the image brighter than previous which helps better for segmentation. Other values rather than 25 makes the image too much brighter which can cause inappropriate segmentation of tumor region Segmentation of the tumor region was carried out using OTSU's Thresholding[16]. The enhanced image was then converted to binary image using Otsu's global thresholding method. In this method it deals with two set of pixels' variance which is background and foreground. A threshold value was searched that minimizes the intra-class variance (the variance within the class), defined as a weighted sum of variances of the two classes: 


$$
\sigma_{\mathrm{w}}^{2}(\mathrm{t})=\mathrm{w}_{0}(\mathrm{t}) \sigma_{0}^{2}(\mathrm{t})+\mathrm{w}_{1}(\mathrm{t}) \sigma_{1}^{2}(\mathrm{t})
$$

Weights $w_{0,1}$ are the probabilities of the two classes separated by a threshold $t$ and $\sigma_{0,1}^{2}$ are variances of these two classes. The class probability $w_{0,1}(t)$ is completed from $\mathrm{L}$ histograms:

$$
\begin{aligned}
& \mathrm{w}_{0}(\mathrm{t})=\sum_{i=0}^{t-1} p(i) \\
& \mathrm{w}_{1}(\mathrm{t}) \sum_{i=t}^{L-1} p(i)
\end{aligned}
$$

In this method minimizing the intra-class variance is the same as maximizing inter-class variance

$$
\begin{aligned}
& \sigma_{\mathrm{b}}^{2}(\mathrm{t})=\sigma^{2}-\sigma_{\mathrm{w}}^{2}(\mathrm{t})=\mathrm{w}_{0}\left(\mu_{0}-\mu_{\mathrm{T})}{ }^{2}+\right. \\
& \mathrm{w}_{1}\left(\mu_{1}-\mu_{\mathrm{T})}{ }^{2}=\mathrm{w}_{0}(\mathrm{t}) \mathrm{w}_{1}(\mathrm{t})\left[\mu_{0}(t)-\mu_{1}(t)\right]^{2}\right.
\end{aligned}
$$

Which is expressed in terms of class probabilities $w$ and class mean $\mu$.While the class mean $\mu_{0,1, T}(t)$ is:

$$
\begin{aligned}
& \mu_{0}(t)=\sum_{i=0}^{t-1} i p(i) / \mathrm{w}_{0} \\
& \mu_{1}(t)=\sum_{i=t}^{L-1} i p(i) / \mathrm{w}_{1} \\
& \mu_{T}=\sum_{i=0}^{L-1} i p(i)
\end{aligned}
$$

For optimal selection of threshold value, 0.3 were added with global threshold value that provides better segmentation of tumor region. Any value within the range 0 to 1 rather than this causes inaccurate segmentation. Fig. 8(c) shows optimal selection of additional value and 8(b), 8(d), 8(e) shows wrong selection Morphological erosion and dilation was applied respectively with an structuring element of $[1 ; 1 ; 1 ; 1]$ which provides better segmentation showing in Fig. 5(e) and 5(f).The holes were filled and the blob was separated after opening the biggest blob by removing pixels smaller than 250 from the hole filled image which provides the exact segmentation of tumor region. Fig. 5(g) and 5(h) shows hole filling and biggest blob opening respectively. Tumor in blob is detected by invoking MATLAB function: regionProps (binaryImage,'BoundingBox') to find object properties in the binary image showing in Fig. 5(i). Global Thresholding (Otsu's method) [19] was used to extract the region of tumor. The main advantage is the simplicity of calculation of the threshold. As well as it takes less calculation time to select a threshold value for segmentation. Procedural diagram of TMP were shown in Fig. 4 and Fig. 5 shows the Procedural outputs of Thresholding and Morphological Processing on BRATS_HG0001_FLAIR image.

Algorithm-2: Thresholding and Morphological Processing Input: BRATS_HG_0001_FLAIR MRI

\section{Begin:}

1. Load the input image.

2. Convert input image into grayscale image.

3. Enhance the gray image Applying Median filter.

4. Convert enhanced image into Binary image by thresholding.

5. Erode the binary image with Structuring element

$$
[1 ; 1 ; 1 ; 1]
$$

6. Dilate the image after eroding with Structuring element $[1 ; 1 ; 1 ; 1]$
7. Fill the holes of dilated image.

8. Open the biggest blob after hole filling.

9. Detect Tumor with blob after finding 4 points of rectangle.

End;

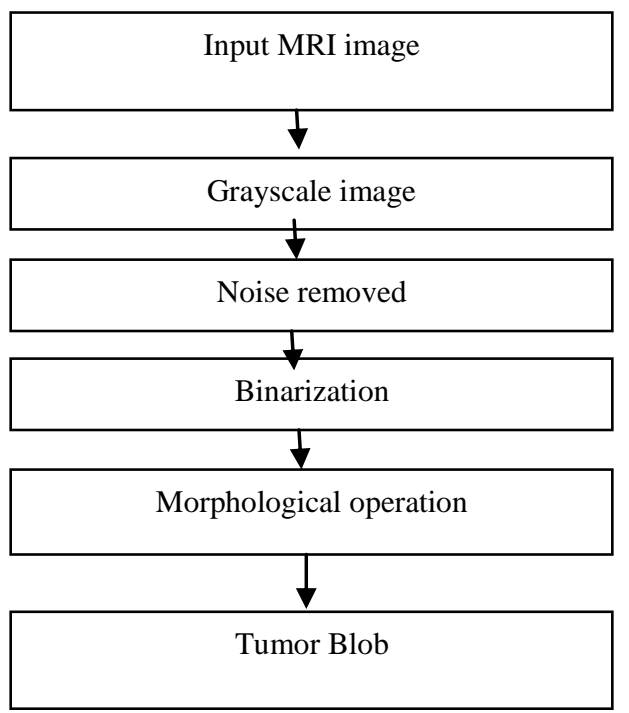

Fig 4: Diagram of Thresholding and Morphological Processing.

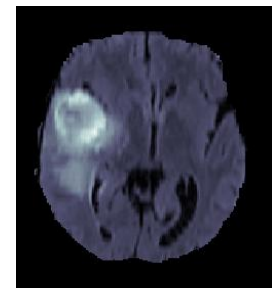

a)Input image

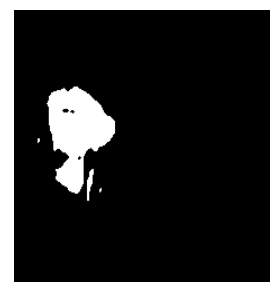

d)Binarization

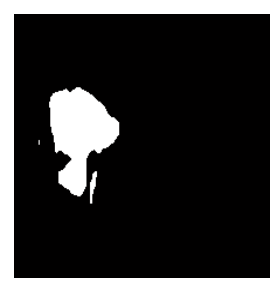

g)Filling holes

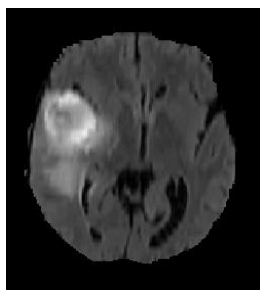

b)Grayscale image

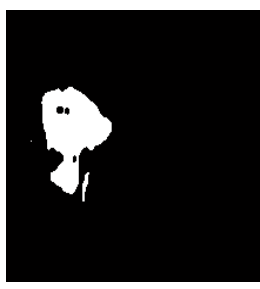

e)Eroding by 4 neighborhood

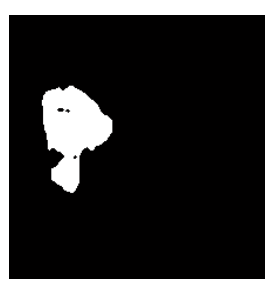

h)Biggest blob opening

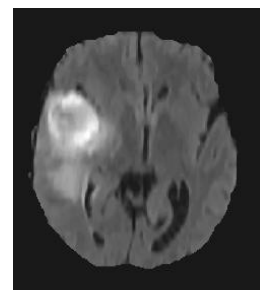

c)Noise removed

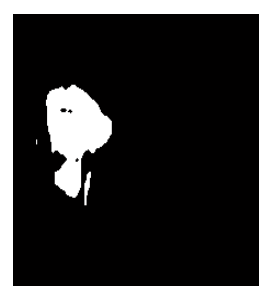

f)Dilation by 4 neighborhood

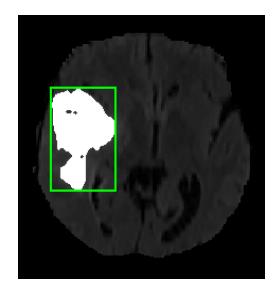

i)Tumor blob
Fig 5: Procedural output of threshold segmentation and morphological processing 


\section{RESULT ANALYSIS \& DISCUSSION}

\subsection{BRATS Database}

The proposed study was implemented using BRATS brain Tumor database that contains 72 patients' MRI, each with 4 modalities (FLAIR, T1, T1C, and T2). Brain Tumor the FLAIR MRI modality allows a better detection due to bright signal of the CSF (Cerebrospinal fluid) is suppressed in it [1]. Our research was implemented using 72 Flair images of 72 Patients. Fig. 6 shows 4 types of common MRI modalities.

\subsection{Noise Removing}

The Fig. 7(b) and 7(c) shows the effect of noise removing when it was in binary image. Salt and pepper noise were removed by applying median filter [17] and addition of pixel value 25 with each image pixels provides better result at the step of threshold segmentation showing in Fig. 7(c).

\subsection{Thresholding}

Tumor segmentation was performed using OTSU's Global thresholding $(\mathrm{T})$ value and adding 0.3 with $\mathrm{T}$. Poor selection of threshold value were shown in Fig. 8(b) while optimal selection of threshold value were shown in Fig. 8(c) but Fig. 8(d) and 8(e) shows tumor segmented region with losing details.

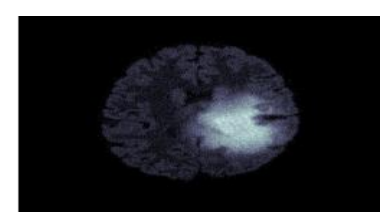

a)FLAIR

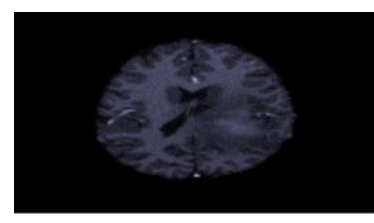

c) T1C

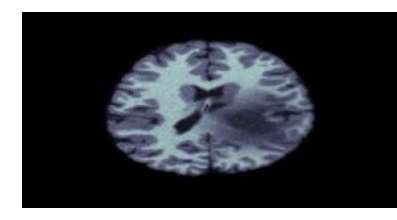

b)T1

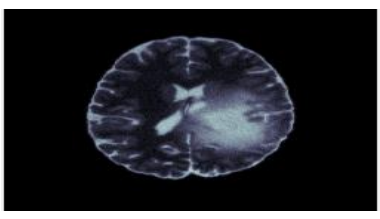

d)T2

Fig 6: Common MRI Modalities in Medical Imaging

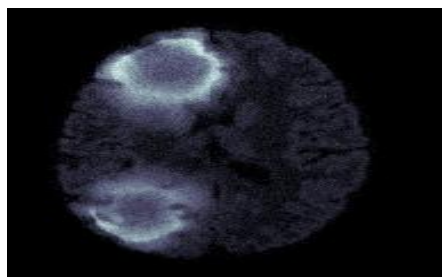

a)SimBrats_HG0014_FLAIR

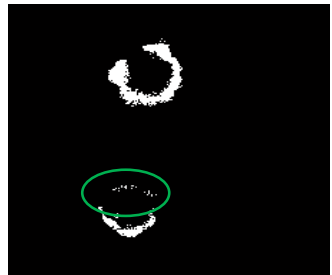

b) Before removing noise

Fig 7: Effect of Noise Removing
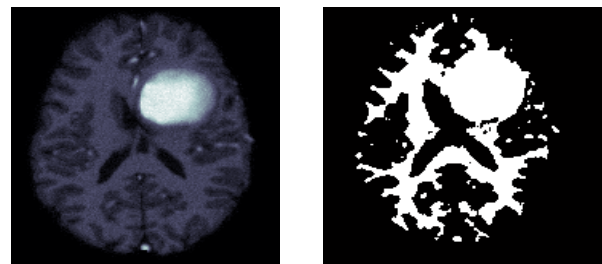

a)SimBrats_HG0001_FLAIR

b) $\mathrm{T}+0.1$

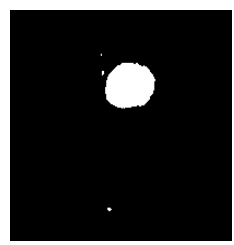

c) $\mathrm{T}+0.3$

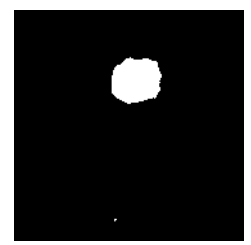

d) $\mathrm{T}+0.5$

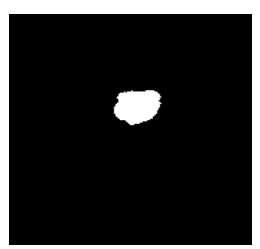

e) $\mathrm{T}+0.7$
Fig 8: Effect of image Binarization

\subsection{Image Morphology}

Perfect localization of tumor affected area in human brain was found by morphological image processing. Morphological erosion, dilation, hole filling, biggest blob opening was applied in our study showing in Fig. 10(b), (c), (d), (e) respectively which results in accurate detection of tumor region showed in Fig. 10(f). False detection results were found without applying any morphological image processing showing in Fig. 9.

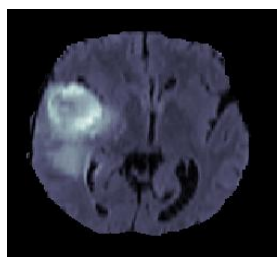

a) Input

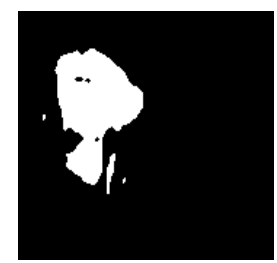

b)Without morphology

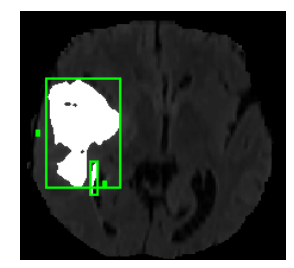

c) False detection
Fig 9: False detection without Morphological Processing

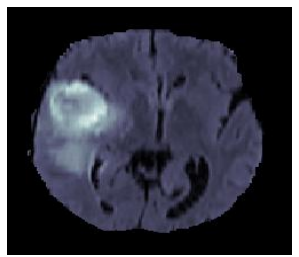

a)Input image

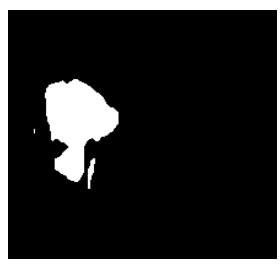

d)Hole filling

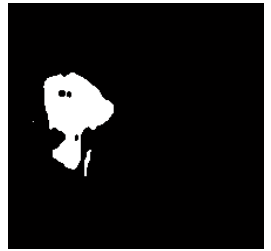

b)Eroding

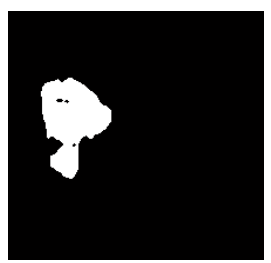

e)Biggest blob opening

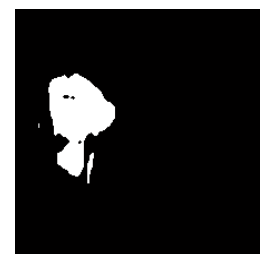

c)Dilating

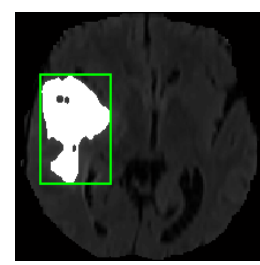

f)Accurate detection
Fig 10: Accurate Detection with Applying Morphological Processing 


\subsection{Output of Cross Validation}

38 patients Tumor as True positive (TP) were found by applying Color based segmentation using k-means(CBS) method while Thresholding and Morphological Processing(TMP) method finds 61 patients Tumor as True positive Result of tumor detection by CBS method and the result of TMP were shown in table 1.

The proposed method were also analyzed the true positive output performance of CBS and TMP method using 4 types of MRI modalities where 72 of each modality have been used. Experimental results showed 38,7,0,0 true positive value using FLAIR,T1C,T1,T2 MRI respectively applying CBS method and 61,11,0,9 true positive value applying proposed(TMP) method. True positive output comparison with CBS and TMP method were shown in Fig. 11 and aaccuracy in Tumor detection by CBS \& TMP were shown in Fig. 12.Optimal selection of number of cluster $k=3$ for CBS method were shown in table 3 and experimental outputs of TMP methods were shown in table 4 .

The proposed method gives a strong response for detecting of multiple tumor regions in a single MRI slice as well as smallest region of tumor showing in Fig. 13(a) and 13(b) respectively, though it is difficult to detect by medical expertise and also impossible by other existing method .Performance and comparison analysis of proposed method using BRATS brain tumor dataset were shown in table 5 where various existing methods were developed in the Multimodal Brain Tumor image segmentation Benchmarks (BRATS) [18].

\begin{tabular}{|c|c|c|}
\hline \multicolumn{2}{|c|}{ Table 1: Result of tumor detection } \\
\hline Output & $\begin{array}{c}\text { CBS method } \\
\text { Rate(\%) }\end{array}$ & $\begin{array}{c}\text { TMP method } \\
\text { Rate ( \%) }\end{array}$ \\
\hline True positive & 52.78 & 84.72 \\
True negative & 25 & 11.11 \\
False positive & 0 & 1.39 \\
False negative & 22.22 & 2.78 \\
\hline
\end{tabular}

\begin{tabular}{|c|c|c|}
\hline \multicolumn{2}{|c|}{ Table 2: Detection and Extraction time for this work } \\
\hline Input image & \multicolumn{2}{|c|}{ Elapsed time in second } \\
\cline { 2 - 3 } & CBS & TMP \\
\hline BRATS_HG0001_FLAIR & 6.5 & 3.7 \\
BRATS_HG0004_FLAIR & 7.1 & 3.6 \\
BRATS_HG0006_FLAIR & 7.0 & 3.3 \\
BRATS_HG0022_FLAIR & 6.6 & 3.3 \\
BRATS_LG0002_FLAIR & 7.1 & 3.3 \\
SimBRATS_HG0003_FLAIR & 6.4 & 4.1 \\
SimBRATS_HG0007_FLAIR & 6.6 & 3.8 \\
SimBRATS_HG0022_FLAIR & 6.7 & 3.7 \\
SimBRATS_LG0003_FLAIR & 6.4 & 3.7 \\
SimBRATS_LG0011_FLAIR & 6.6 & 3.5 \\
\hline Average detection time & 6.7 & 3.6 \\
\hline
\end{tabular}

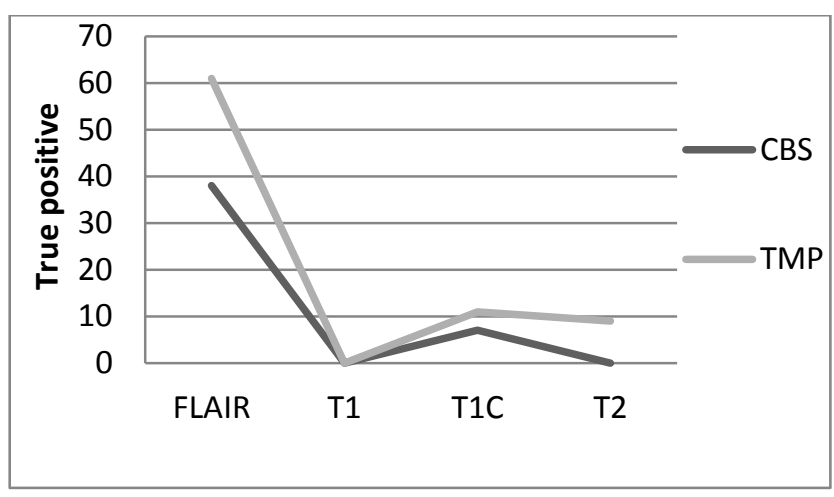

Fig 11: True Positive Output comparison with CBS and TMP

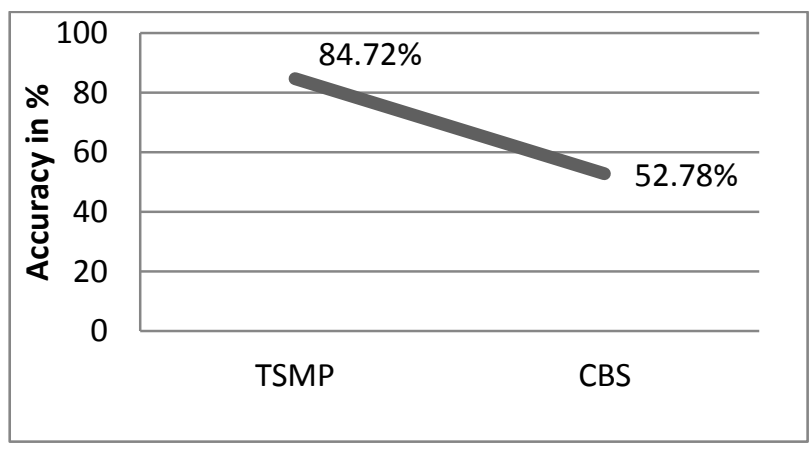

Fig 12: Accuracy in Tumor Detection by CBS \& TMP 


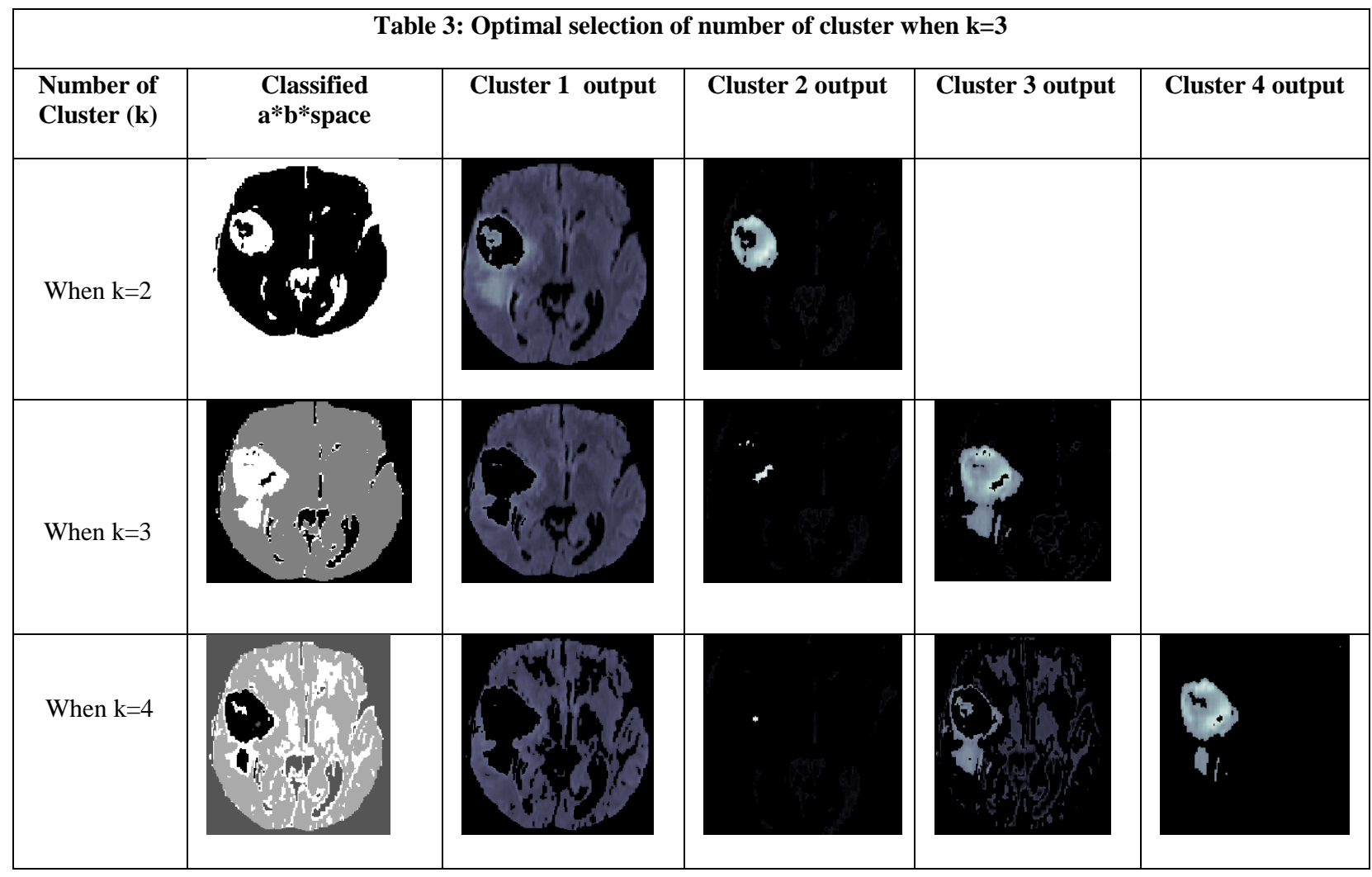

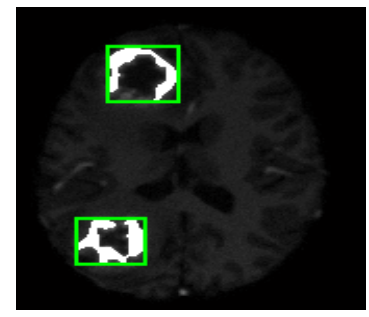

a)SimBrats_HG0014_FLAI $\mathrm{R}$

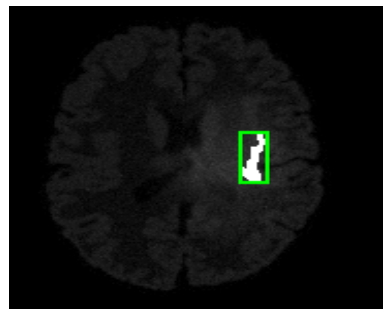

b)SimBrats_LG0005_FLAIR

Fig 13: Tumor with Multiple region and smallest region

Applying Color based image segmentation using k-means clustering finds an accuracy rate of $52.78 \%$ in detecting brain tumor while thresholding segmentation and morphological processing method provides an accuracy rate of $84.72 \%$ detecting 61 patients Tumor from 72 different patients MRI images using only FLAIR modalities. 


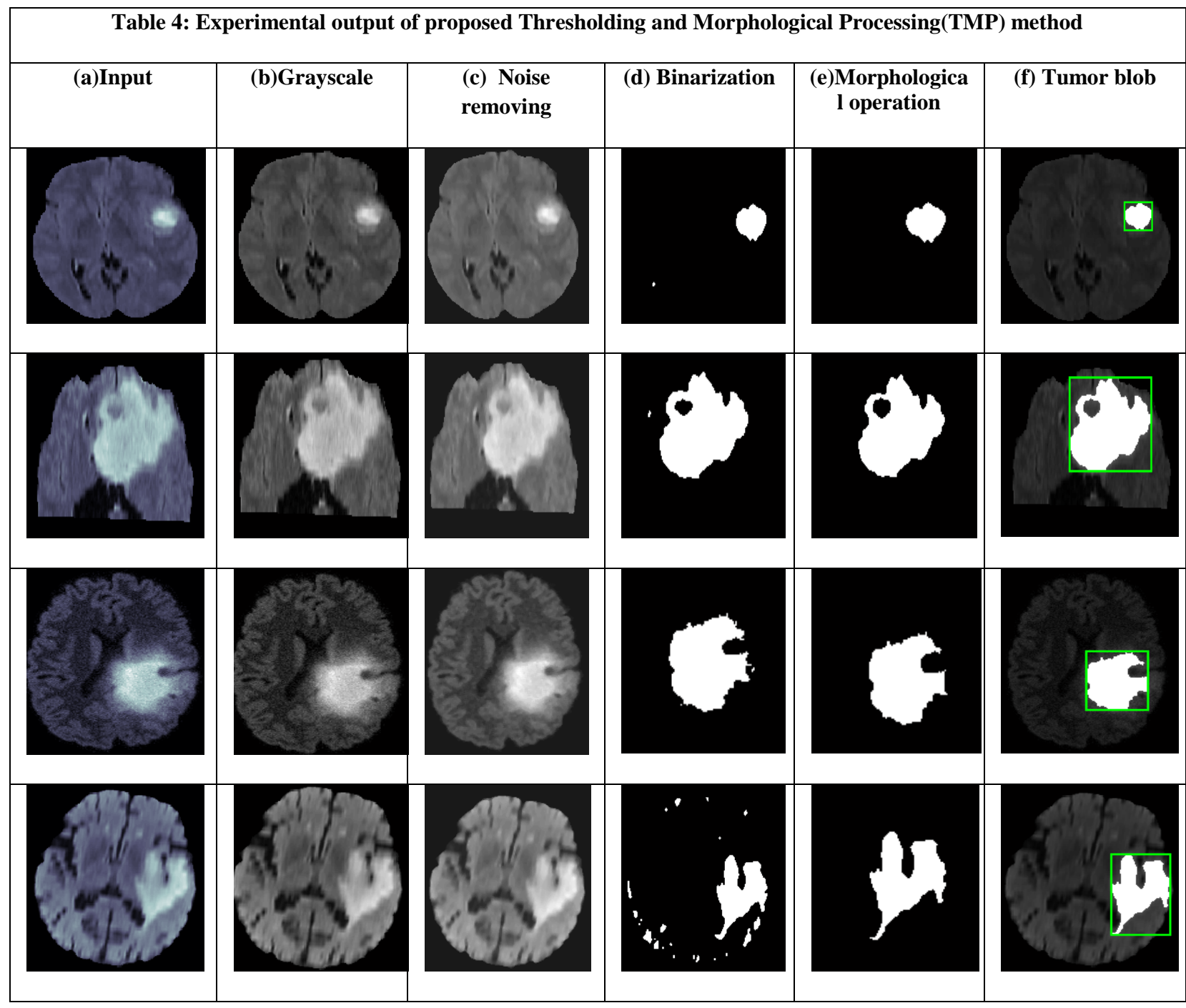

\begin{tabular}{|c|c|c|c|}
\hline \multicolumn{3}{|c|}{ Table-5: Performance and comparison analysis of proposed method BRATS brain tumor dataset } \\
\hline Algorithm & MRI modalities & Approach & Accuracy rate \\
\hline Corso 2008 & T1,T1C,T2,FLAIR & Weighted aggregation & $34-69 \%$ \\
Verma 2008 & T1,T1C,T2,FLAIR,DTI & SVM & $78 \%$ \\
Wels 2008 & T1,T1C,T2 & Discriminative model w/CRF & $50-75 \%$ \\
Cobzas 2009 & T1C,FLAIR & Level set w/CRF & $60-70 \%$ \\
Wang 2009 & T1C & Fluid vector flow & $40-70 \%$ \\
Menze 2010 & T1,T1C,T2,FLAIR & Thresholding and morphological & $84.72 \%$ \\
Proposed & FLAIR & processing & \\
\hline
\end{tabular}

\section{CONCLUSION}

This paper presents a new and efficient method for brain tumor detection from patients MRI by thresholding and morphological processing. A set of preprocessing steps including image morphology, contrast enhancement and noise filtering techniques have been applied to detect tumor region. The preprocessing segmentation processes reduce noise and accurately detect region of interest (ROI) for brain tumor. The objective of this work is to develop a CAD system and improve the existing system for automated brain tumor 
detection. The performance was evaluated using a set of 72 FLAIR MRI of 72 patients from BRATS brain tumor database where 61 patients tumor have been detected accurately. From the computational point of view, the detection step is less time consuming. Running time of the program is about 3.6 seconds on Intel Pentium Dual Core 2.40GHz CPU with 1.86 GB. The computational costs was acceptable since the detection rate were good. The success rate of our proposed algorithm indicates $84.72 \%$ true positive, $1.39 \%$ false positive and $2.78 \%$ false negative results. The implementation and qualitative evaluation of experimental results was carried out using MATLAB R2014a.lnk on 32 bit Windows 8 operating system. The accuracy rate is very much promising to detect brain tumor from patients MRI and it can be used to classify the types of tumor according to medical diagnosis system.

\section{REFERENCES}

[1] Olaf Dietrich "Brain MRI image types", LudwigMaximilian-University of Munich.

[2] S. N. Sulaiman, S. M. Che Ishak, I. Isa, N. Hamzah : "De-noising of Noisy MRI Brain Image Using The Switching-based Clustering Algorithm", IEEE International Conference on Control System, Computing and Engineering(ICCSCE), 28 - 30 November 2014, Penang, Malaysia.

[3] N. Sachin:"brain tumor detection based on bilateral symmetry information", International Journal of Engineering Research and Applications( IJERA),ISSN: 2248-9622.

[4] http://www.med.harvad.edu/AANLIB/home.html,access ed on 8 August 2013.

[5] M. K. Kowari,S. Yadav.2012: "Brain Tumor Detection and Segmentation using Histogram Thresholding", International Journal of Engineering and Advanced Technology(IJEAT) ISSN: 2249-898, Volume-1, Issue4, Journal, India.

[6] M. M. Ahmed, D. Bin Mohamad: "Segmentation of Brain MR Images for Tumor Extraction by Combining K-means Clustering and Perona-Malik Anisotropic Diffusion model.International Journal of Image Processing, Volume(2): Issue(1).

[7] Nagalkaar V.J, Asole S.S.2012: "Brain Tumor Detection using Digital Image Processing based on Soft
Computing," Journal of Signal and Image Processing, Volume 3, Issue 3, Issn: 0976-8882.

[8] P. Vasuda, S. Satheesh.2010: "Improved Fuzzy C-Means Algorithm for MR Brain Image Segmentation", International Journal on Computer Science and Engineering (IJCSE), vol. 02, no.05, pp 1713-1715.

[9] Prastawa M, Bullitt E, Ho S, Gerig G.2004: “A brain tumor segmentation framework based on outlier detection", Medical Image Analysis.

[10] Zhou J, Chan K. L, Chongand VFH, Krishnan SM.2005: "Extraction of brain tumor from MR images using oneclass support vector machine ", Conf Proc IEEE Eng Med Bio Soc. 2005;6:6411-4.

[11] Y. Sun, B. Bhanu, S. Bhanu.2009: "Automatic Symmetry-integrated Brain Injury Detection in MRI Sequences", Conference, Proc of the International Conference on Image Processing, ICIP 2009, 7-10 November 2009, Cairo, Egypt.

[12] S. Zin Oo, Aung Soe Khaing "brain tumor detection and segmentation using watershed segmentation and morphological operation”, IJRET; ISSN: 2319-1163.

[13] http://challenge.kitware.com/midas/folder/102,accessed on 10 November 2015.

[14] B. Menze, A. Jakab, S. Bauer, M. Reyes, M. Prastawa, and K. Van Leemput, "MICCAI 2012 Challenge on Multimodal Brain Tumor Segmentation".

[15] http://www.mathworks.com/matlabcentral/fileexchange/ 29067-look3d--a-3-d-image-viewer,accessed on 15 November 2015.

[16] Otsu, N., "A Threshold Selection Method from GrayLevel Histograms," IEEE Transactions on Systems, Man, and Cybernetics, Vol. 9, No. 1, 1979, pp. 62-66.

[17] Van Solihin and C. G. Leedham "The Multi-stage Approach to Grey-Scale Image Thresholding for Specific Applications".

[18] The Multimodal Brain Tumor Image Segmentation Benchmark (BRATS); IEEE Transaction on Medical Imaging, Vol. 34, No. 10, October 2015. 